Article

\title{
Effects of Modifying Acidity and Reducibility on the Activity of NaY Zeolite in the Oxidative Dehydrogenation of $n$-Octane
}

\author{
Siyabonga S. Ndlela ${ }^{1}{ }^{(}$, Holger B. Friedrich ${ }^{1, * \mathbb{C}}$ and Mduduzi N. Cele ${ }^{2}$ \\ 1 School of Chemistry and Physics, Westville Campus, University of KwaZulu-Natal, Private Bag X 54001, \\ Durban 4000, South Africa; ndlelas@ukzn.ac.za \\ 2 School of Physical and Chemical Sciences, Mafikeng Campus, North-West University, Private Bag X 2046, \\ Mmabatho 2745, South Africa; Nicholas.Cele@nwu.ac.za \\ * Correspondence: friedric@ukzn.ac.za; Tel.: +27-31-2603107
}

Received: 13 February 2020; Accepted: 6 March 2020; Published: 27 March 2020

\begin{abstract}
Non-coking stable alkaline earth metal $(\mathrm{M}=\mathrm{Mg}$, $\mathrm{Sr}$, and $\mathrm{Ba})$ modified $\mathrm{Ga}-\mathrm{NaY}$ catalysts were prepared by ionic-exchange and tested in oxidative dehydrogenation (ODH) of $n$-octane using air as the source of oxygen. The role of the alkaline earth metals in NaY was to poison the acid sites while enhancing the basic sites responsible for ODH. The exception was the calcium modified NaY, which was more acidic than the parent NaY, coking and unstable under the ODH conditions used in this study. The role of gallium was to enhance the dehydrogenation pathway and improve the stability of NaY. The sequence of increasing selectivity to octenes followed the order: $\mathrm{CaGa}-\mathrm{NaY}<$ Ga-NaY $<$ MgGa-NaY $<$ SrGa-NaY < BaGa-NaY. The highest octene selectivity obtained was 37\% at iso-conversion of $6 \pm 1 \%$ when $\mathrm{BaGa}-\mathrm{NaY}$ was used at a temperature of $450{ }^{\circ} \mathrm{C}$. The activity of the catalysts was directly proportional to the reducibility of the catalysts, which is in agreement with expectations.
\end{abstract}

Keywords: oxidative dehydrogenation; $n$-octane; Ga-NaY; ionic exchange; acidity

\section{Introduction}

Oxidation, cracking, dehydrogenation, dehydrocyclization, and oxidative dehydrogenation are possible reactions that can activate paraffins using heterogeneous catalysts. Dehydrogenation $(\mathrm{DH})$ of $n$-octane is one of the important reactions industrially and can be carried out using a variety of catalysts, both mono- and bi-functional [1-4]. Paraffins are easily and cheaply obtained from natural gas and petroleum, and this, with their relative environmental friendliness, makes them interesting alternatives to olefinic and aromatic feedstocks. Furthermore, the conversion of alkanes to alkenes and aromatics, which are important raw materials in the petrochemical and detergent industries, is of importance academically and industrially [5-7].

$\mathrm{DH}$ reactions are characterized by their low conversions due to thermodynamic equilibrium limitations, endothermic nature and high temperature operating conditions ranging above $450{ }^{\circ} \mathrm{C}$ [8]. Catalysts under DH reactions are likely to deactivate since the catalyst is prone to coke deposition on the active sites. This subsequently leads to lower yields of alkenes. More recent developments have led to the introduction of oxygen to the DH reaction, which rendered the process exothermic, with much reduced thermodynamic limitations and reduced coke deposition on the catalyst. This is the reason why oxidative dehydrogenation $(\mathrm{ODH})$ would be the preferred reaction in the petrochemical industry $[9,10]$. Very high COx production remains the major disadvantage of ODH reactions. 
A number of systems have been used previously for the ODH of alkanes $[9,11]$. For example, alkaline earth metal oxide-based catalysts promoted by other elements such as lithium and chlorine showed improved oxidative dehydrogenation activity [12,13].

Rare earth metal oxide containing catalysts are used in ODH reactions because of their high thermal stability and their basicity. $\mathrm{Sm}, \mathrm{Nd}, \mathrm{Ce}, \mathrm{La}, \mathrm{Pr}$, and $\mathrm{Tb}$ are among the commonly studied rare earth metals in the ODH of alkanes. The best conversions and selectivities were reported when these metals were promoted by alkaline earth metals such as Sr and Ba. Using rare earth metals containing catalysts in ODH generally improved activity and reduced deep oxidation [14-16].

So far, the most studied catalysts are the transition metal oxide-based catalysts, with vanadium or molybdenum being the most studied active components. Such catalysts are employed in ODH, with common supports such as $\mathrm{Al}_{2} \mathrm{O}_{3}, \mathrm{TiO}_{2}, \mathrm{ZrO}_{2}, \mathrm{MgO}$, and $\mathrm{SiO}_{2}$. Other studies have also involved the use of supports such as hydrotalcite, rare earth metals, and SBA-15, to mention a few [17-19]. These systems work on the basis of the reducibility of the active metals and the acid base characteristics of the catalyst and have shown interesting results. The reaction mechanism associated with this type of catalyst is the Mars-van Krevelen mechanism, which involves the abstraction of lattice oxygen by the alkane to form the corresponding alkene and water.

The limitations of these mentioned systems, which include low surface area leading to active metal agglomeration, low thermal stability, and complicated synthesis procedures which can hinder reproducibility, leave considerable room for improvement. Among other systems are zeolite materials with some interesting properties such as good thermal stability, high surface area, and ease of synthesis and modification. A few studies in recent years have focused on the ODH of alkanes using different zeolite materials [20-22]. In one of the most recent studies, the role of gallium in ODH of a short chain alkane was investigated [23]. In this study, we introduce developments in ODH of $n$-octane using the $\mathrm{NaY}$ zeolite modified with gallium in conjunction with alkaline earth metals, specifically looking at the influence on the acidity and reducibility which in turn affects the activity and stability of the catalyst.

\section{Results and Discussion}

\subsection{Catalysts Characterization}

\subsubsection{Powder XRD}

X-ray powder diffraction (XRD) diffractograms of 0.02BaGa-NaY, 0.08BaGa-NaY, NaY, Ga-NaY, MgGa-NaY, CaGa-NaY, SrGa-NaY and BaGa-NaY, shown in Figure 1, depict the characteristic peaks from the faujasite group framework [24-26]. This implies that the introduction of alkaline earth metals and calcination of the zeolite does not adversely affect the framework of the faujasite zeolite. The similarities between the parent $\mathrm{NaY}$ and the modified zeolites diffractograms indicate that the degree of crystallinity (see SI) of the material does not change appreciably after the modification. All the modified zeolites showed only the NaY phase with the absence of gallium oxide peaks, which was attributed to the small amount of gallium used and good dispersion [27].

\subsubsection{FT-IR}

Figure 2 shows the Fourier-transform infrared spectroscopy (FT-IR) spectra of all the prepared catalysts. Upon the introduction of Ga and alkali earth metals, there was a shift in the bands compared to the parent NaY. All the bands at around 463 and $551 \mathrm{~cm}^{-1}$ are due to $\mathrm{T}-\mathrm{O}(\mathrm{T}=\mathrm{Al} / \mathrm{Si})$ bending vibrations of internal and external tetrahedra in the zeolite respectively $[25,28]$. Bands around $759 \mathrm{~cm}^{-1}$ are assigned to the symmetric stretching of the external linkage and internal tetrahedra respectively $[25,28]$. The intense band at about $1003 \mathrm{~cm}^{-1}$ is due to Si-O bending, and at about $1654 \mathrm{~cm}^{-1}$, the $\mathrm{H}-\mathrm{O}-\mathrm{H}$ symmetric stretching band appears [28]. The bands, at around $3000 \mathrm{~cm}^{-1}$ are attributed to stretching vibrations of hydroxyl groups of water molecules in the pores of the zeolites [29-31]. 


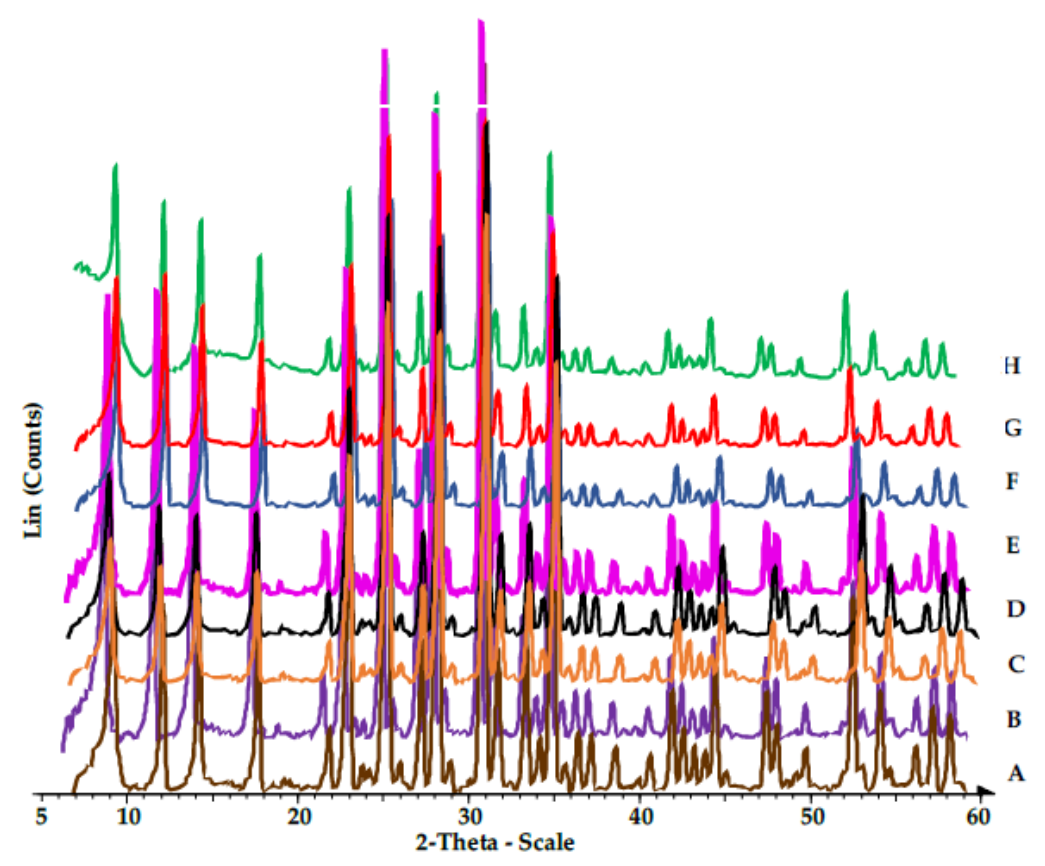

Figure 1. XRD diffractograms of (A) NaY, (B) Ga-NaY, (C) 0.02BaGa-NaY, (D) MgGa-NaY, (E) CaGa-NaY, (F) SrGa-NaY, (G) BaGa-NaY, (H) 0.08BaGa-NaY.

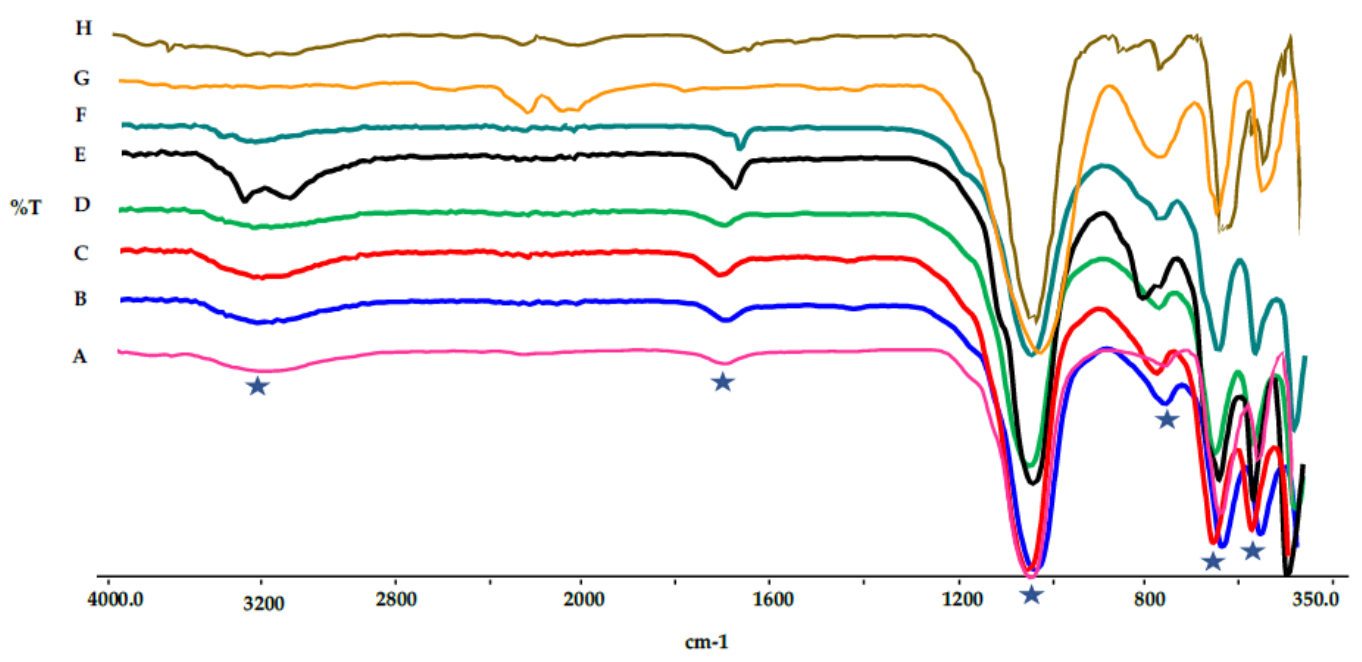

Figure 2. FT-IR spectra of (A) NaY, (B) Ga-NaY, (C) 0.02BaGa-NaY, (D) MgGa-NaY, (E) CaGa-NaY, (F) SrGa-NaY, (G) BaGa-NaY, (H) 0.08BaGa-NaY.

The different intensities and the shifts of the bands are attributed to the different alkaline earth metals introduced into the extra-framework of the NaY material. The stretching vibration band of the hydroxyl group of the water molecule of $\mathrm{CaGa}-\mathrm{NaY}$ is split, and this may be attributed to the heterogeneity of the hydroxyl group as a result of silica-hydroxyl groups of various bridge geometries resulting in the presence of hydroxyls of various acid strengths [32]. BaGa-NaY showed the weakest intensity of the $\mathrm{Si}-\mathrm{OH}$ band which is also associated with the weak Brønsted acid sites of the catalyst.

\subsubsection{ICP-OES Studies}

Inductively coupled plasma atomic emission spectroscopy (ICP-OES) analysis results shown in Table 1 confirmed the successful ionic exchange procedure and showed that Na decreased in the zeolite with the introduction of gallium and the alkaline earth metals. About one weight percent of gallium 
was incorporated in all the samples with about $1.5 \mathrm{wt} \%$ of alkaline earth metals. All the compared catalysts had about 10 wt \% of Na left in the extra-framework.

Table 1. ICP-OES metal ratios of the fresh NaY catalysts.

\begin{tabular}{ccc}
\hline Catalysts & Ga (wt \%) & Alkali Metal (wt \%) \\
\hline GaNaY & 1.4 & - \\
CaGa-NaY & 1.1 & 1.7 \\
MgGa-NaY & 0.88 & 1.5 \\
SrGa-NaY & 1.0 & 1.5 \\
BaGa-NaY & 1.2 & 1.4 \\
\hline
\end{tabular}

\subsubsection{Temperature Programmed Desorption with $\mathrm{NH}_{3}$ and $\mathrm{CO}_{2}$}

For ammonia TPD (Table 2$)$, the low temperature $\left(\leq 200^{\circ} \mathrm{C}\right)$ ammonia desorption corresponds to the desorption of loosely bound ammonia, high temperature $\left(\geq 400{ }^{\circ} \mathrm{C}\right)$ ammonia desorption corresponds to strong acid sites, and the intermediate corresponds to weak and medium strength acid sites [33]. NaY is known for its weak acid sites due to the high density of aluminium in its framework. This was consistent with our findings [34]. The introduction of gallium introduced medium strength acid sites, arising from the migration of some aluminium from the framework to extra-framework positions following ionic exchange and calcination at high temperatures. This led to an increase in the intrinsic acidity of the zeolite [35]. The strong acid sites increased upon the introduction of alkaline earth metals and this could be attributed to strong Lewis acid sites present from the bare cations in $\mathrm{NaY}$, this is in line with the pyridine FT-IR analysis (Section 2.1.6), which showed that at $200{ }^{\circ} \mathrm{C}$, strong Brønsted acid sites are absent in the alkaline earth metal modified GaNaY. This was not the case for CaGa-NaY, which has both Lewis and Brønsted acid sites, as the Brønsted acid sites band was retained in the pyridine FT-IR spectrum. Due to the weaker interaction between ammonia and alkaline earth metals, the total acidity of the prepared catalysts decreased with the introduction of alkaline earth metals [36]. Calcium is hygroscopic in nature, and therefore easily absorbs water which forms the hydroxyl groups responsible for the pronounced Brønsted acidity shown by CaGa-NaY. NH $\mathrm{N}_{3}-\mathrm{TPD}$ profiles (Figure S3) also show that the acid sites at high temperature ammonia adsorption have an overlap of peaks which is an indication of the presence of both the Lewis and Brønsted acid sites, with the former adsorbing at higher temperatures [37-39].

Table 2. Acid properties of different zeolites studied by $\mathrm{NH}_{3}$-TPD.

\begin{tabular}{ccccc}
\hline Zeolite & $\mathbf{W}\left(\mathbf{2 0 0}{ }^{\circ} \mathbf{C}-\mathbf{3 0 0}{ }^{\circ} \mathbf{C}\right)$ & $\begin{array}{c}\text { Acidity Amount }(\boldsymbol{M m o l ~ g} \mathbf{~} \mathbf{~}) \\
\left(\mathbf{3 0 0}{ }^{\circ} \mathbf{C}-\mathbf{4 0 0}{ }^{\circ} \mathbf{C}\right)\end{array}$ & $\mathbf{S}\left(\mathbf{4 0 0}{ }^{\circ} \mathbf{C}-\mathbf{6 0 0}{ }^{\circ} \mathbf{C}\right)$ & Total Acidity \\
\hline NaY & 30.9 & 0 & 0 & 30.9 \\
Ga-NaY & 21.0 & 31.6 & 0 & 52.6 \\
BaGa-NaY & 10.2 & 3.7 & 1.3 & 15.2 \\
MgGa-NaY & 10.3 & 11.8 & 4.3 & 26.4 \\
SrGa-NaY & 4.8 & 8.8 & 10.2 & 23.8 \\
CaGa-NaY & 19.9 & 14.5 & 7.0 & 41.4 \\
\hline
\end{tabular}

$\mathrm{CO}_{2}$-TPD analysis was done to quantify the basic sites of the modified catalysts. The $\mathrm{CO}_{2}$ desorption at 50-160, 160-400, and 550-700 ${ }^{\circ} \mathrm{C}$ corresponded to weak, moderate and strong basic sites, respectively $[30,40]$. The results in Table 3 show that the total amount of basic sites increased with the introduction of the alkaline earth metals besides calcium. Barium, with the lowest electronegativity, had the most basic sites and also had the highest amount of strong basic sites [36,41]. There was no big change between the total acidity of the prepared catalysts. The closeness of the total basicity is relative to the alkaline earth metals used, which all of them belong to the same group and contain 
the same charge, however, there is a noticeable change in basicity as you go down the periodic table, with barium showing more basicity which is consistent with our findings.

Table 3. Base properties of different zeolites studied by $\mathrm{CO}_{2}$-TPD.

\begin{tabular}{ccccc}
\hline & \multicolumn{4}{c}{ Basicity Amount $\left(\mu \mathbf{m o l ~} \mathbf{~}^{-\mathbf{1}}\right)$} \\
Zeolite & $\mathbf{W}\left(\mathbf{0 - 1 6 0}{ }^{\circ} \mathbf{C}\right)$ & $\mathbf{M}\left(\mathbf{1 6 0 - 4 0 0}{ }^{\circ} \mathbf{C}\right)$ & $\mathbf{S}\left(>\mathbf{4 0 0}{ }^{\circ} \mathbf{C}\right)$ & Total Basicity \\
\hline Ga-NaY & 76.5 & 13 & 3 & 92.5 \\
BaGa-NaY & 39 & 6.5 & 74 & 119.5 \\
MgGa-NaY & 66.5 & 23 & 16 & 105.5 \\
SrGa-NaY & 57 & 7.4 & 43 & 107.4 \\
CaGa-NaY & 57 & 5 & 0.4 & 62.4 \\
\hline
\end{tabular}

\subsubsection{Pyridine FT-IR}

Qualitative analysis of the nature of acid sites was carried out by adsorption and desorption of pyridine at 100 and $200{ }^{\circ} \mathrm{C}$. Figure 3 shows the FT-IR spectra of the Ga-NaY modified catalysts in the pyridine vibration range $\left(1400-1700 \mathrm{~cm}^{-1}\right)$. Two bands at 1450 and $1624 \mathrm{~cm}^{-1}$ are related to Lewis-bonded pyridine, two bands at 1400 and $1525 \mathrm{~cm}^{-1}$ are assigned to pyridinium ions $\left(\mathrm{PyH}^{+}\right)$. Physically adsorbed pyridine via hydrogen bonding with surface hydroxyl groups gives absorption bands at 1574 and $1595 \mathrm{~cm}^{-1}$. Lewis and Brønsted acid site interaction contributes to a band at $1489 \mathrm{~cm}^{-1}$ [41]. After treating the samples at $200{ }^{\circ} \mathrm{C}$, (Figure 4) bands associated with Brønsted acid sites disappeared for the alkaline earth metal modified catalysts, with the exception of the Ca modified zeolite. CaGa-NaY contained stronger Brønsted acid sites similar to the unmodified Ga-NaY. This means that the introduction of $\mathrm{Mg}$, $\mathrm{Sr}$, and $\mathrm{Ba}$ increases the strength of Lewis acid sites in the zeolite, which is consistent with the $\mathrm{NH}_{3}$-TPD data.

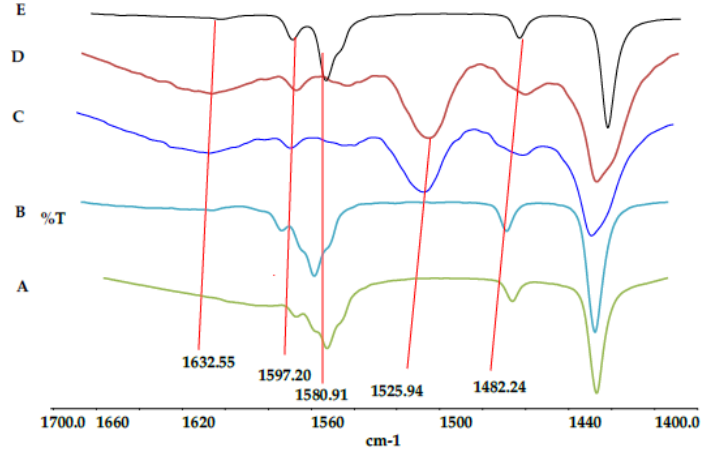

Figure 3. Pyridine-FT-IR of (A) MgGa-NaY, (B) SrGa-NaY, (C) Ga-NaY, (D) CaGa-NaY and (E) BaGa-NaY carried out at $\mathrm{T}=100^{\circ} \mathrm{C}$.

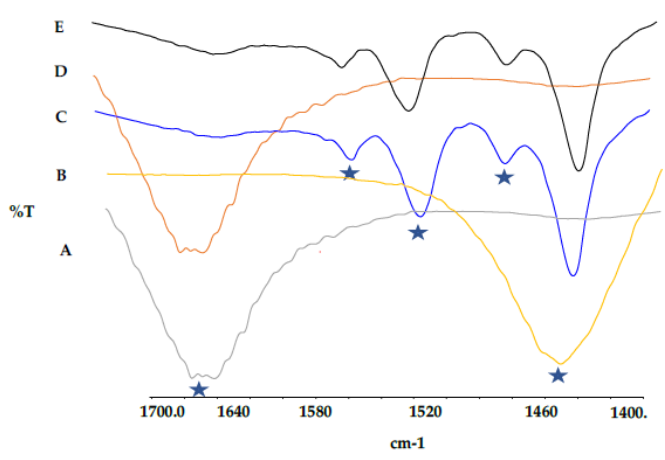

Figure 4. Pyridine-FT-IR of (A) BaGa-NaY, (B) SrGa-NaY, (C) CaGa-NaY, (D) MgGa-NaY and (E) Ga-NaY carried out at $\mathrm{T}=200{ }^{\circ} \mathrm{C}$. 


\subsubsection{Temperature Programmed Reduction}

The redox properties of metal oxides in ODH reactions are one of the key factors in the activation of paraffins. In Figure 5, two reduction peaks are observed for $\mathrm{Ga}-\mathrm{NaY}$ and $\mathrm{CaGa}-\mathrm{NaY}$, and only one reduction peak is observed for $\mathrm{Mg}$, $\mathrm{Sr}$, and $\mathrm{Ba}$ modified $\mathrm{Ga}-\mathrm{NaY}$. The two reduction peaks are attributed to the reduction of $\mathrm{Ga}^{3+}$ to $\mathrm{Ga}^{2+}$ and $\mathrm{Ga}^{1+}$ [42]. In the ODH reaction, $\mathrm{Ga}^{1+}$ is more active then $\mathrm{Ga}^{2+},[41-44]$ hence the single reduction peak for MgGa-NaY, BaGa-NaY, and SrGa-NaY is assigned to the reduction of $\mathrm{Ga}^{3+}$ to $\mathrm{Ga}^{2+}$ since these three catalysts were found to be less active than the other two catalysts with two reduction peaks. Mg and Sr modified Ga-NaY showed peak shoulders at low reduction temperatures, which suggests a slight reduction of $\mathrm{Ga}^{2+}$ to $\mathrm{Ga}^{1+}$. MgGa-NaY had a more significant peak shoulder than SrGa-NaY, which explains the high activity of the Mg modified catalyst compared to the $\mathrm{Sr}$ modified catalyst. The Ba modified catalyst was the least active and had only one reduction peak with no notable peak shoulder. High temperature calcination and modification of the catalysts with alkaline earth metals shifted the reduction peaks to higher temperatures when compared to unmodified Ga-NaY. This may due to three reasons. First is the enhancing effect of alkaline earth metals on metal oxygen bonds in metal oxides, which become more difficult to cleave. The inhibiting effect of alkaline earth metals on $\mathrm{H}_{2}$ activation is a second reason [45]. The third reason might be due to the migration of gallium species to sodalite and hexagonal cages, where reduction is more difficult [46]. Ba and Sr modified Ga-NaY had the highest reduction temperature $\left(\sim 770^{\circ} \mathrm{C}\right)$ and the least consumed moles of hydrogen (Supplementary Table S1).
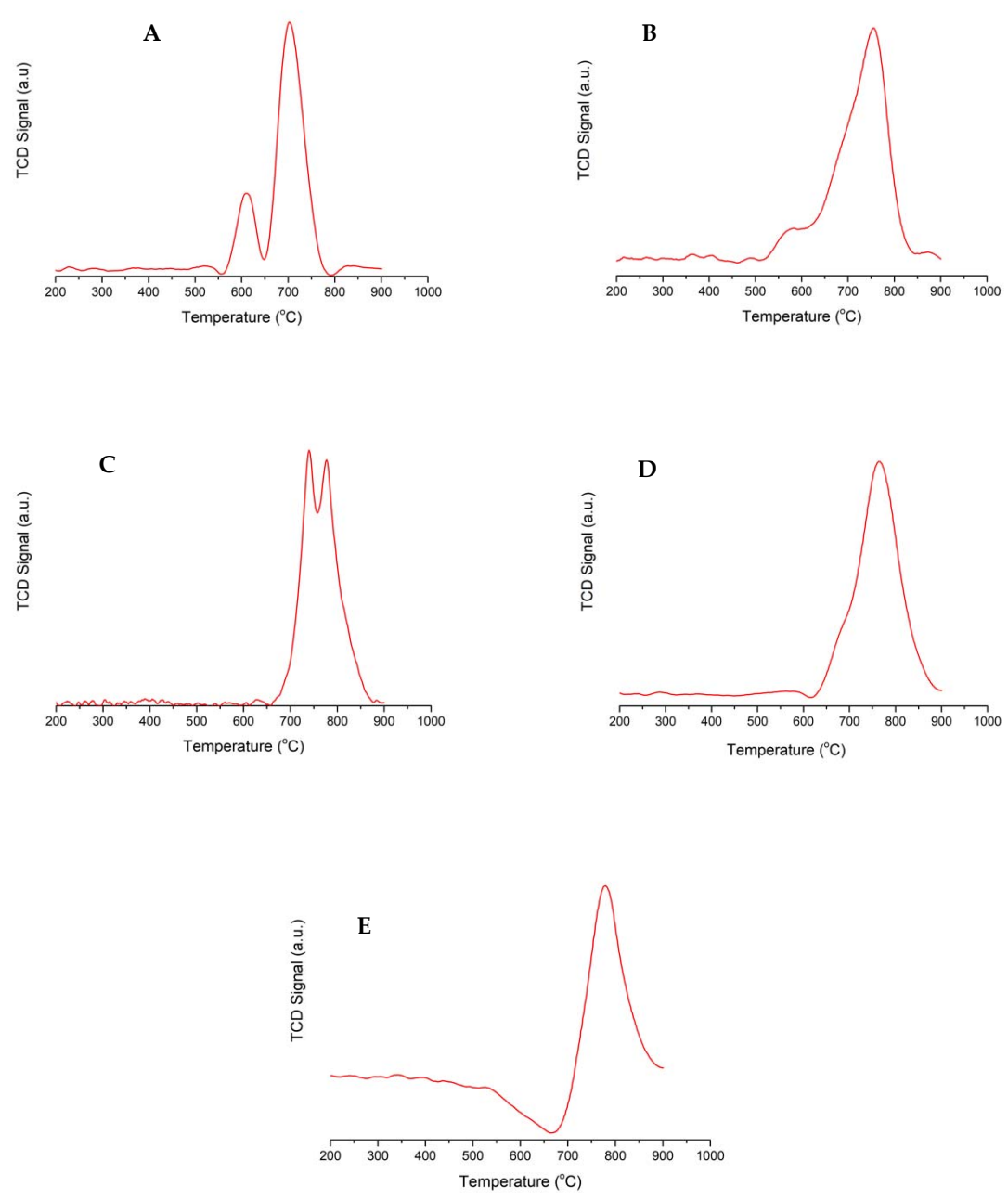

Figure 5. $\mathrm{H}_{2}$-TPR Profiles of (A) Ga-NaY, (B) MgGa-NaY, (C) CaGa-NaY, (D) SrGa-NaY, (E) BaGa-NaY. 


\subsection{Catalytic Performance}

\subsubsection{Ionic Exchange Capacity Study}

To determine the amount of alkaline earth metal that can be efficiently exchanged in Ga-NaY and still give a material with appreciable activity, three catalysts of different Ba concentrations were tested in the activation of $n$-octane. These catalysts were tested under similar reaction conditions, at $\mathrm{T}=450{ }^{\circ} \mathrm{C}$, GHSV $=8000 \mathrm{~h}^{-1}, \mathrm{C}: \mathrm{O} 8: 1$ and $1 \mathrm{~mL}$ of catalyst. The results in Figure 6 show that the conversion increased with decreasing concentration of barium exchanged into the catalysts, which implies that, as the concentration of Ba increases, fewer active sites are accessible on the surface of the zeolite, hence the $0.08 \mathrm{BaGa}-\mathrm{NaY}$ registered the lowest conversion (2.1\%). The selectivity to cracked products and COx is highest over the catalysts with the least concentration of $\mathrm{Ba}(21 \%$ and $29 \%$ respectively). This is because the lower the amount of alkaline metal exchanged, the more acidic the zeolite, and that leads to reduced octene selectivity and more cracked and COx products. The optimum Ba loading was found to be a 0.04 mol loading (based on the octene yield), and all the other catalysts (MgGa-NaY,

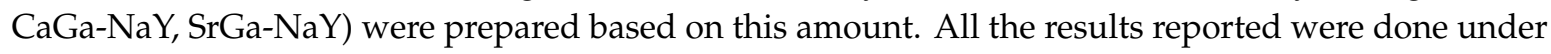
time on stream conditions for three days with sampling every 5-12 hours, and the carbon balance results obtained were all within a $\pm 2 \%$ range of $100 \%$. These results are indicative of the absence of coke formation in the pores of the catalysts, as well as the stability of the alkaline earth metal modified Ga-NaY. CaGa-NaY was an exception to this, as it showed a decrease in activity with time. Therefore, apart from decreasing the acid density of the catalyst, $\mathrm{Mg}$, $\mathrm{Sr}$, and Ba modified $\mathrm{Ga}-\mathrm{NaY}$ also has pores of sufficient size to allow the coke intermediates (alkenes) to pass through without reacting further to form coke deposits that can deactivate the catalyst (Section 2.2.2). However, strong Brønsted acidity associated with CaGa-NaY leads to the strong adsorption of the alkenes on the catalyst surface, which results in coke formation leading to their deactivation. This was also the case for the unmodified parent Ga-NaY catalyst.

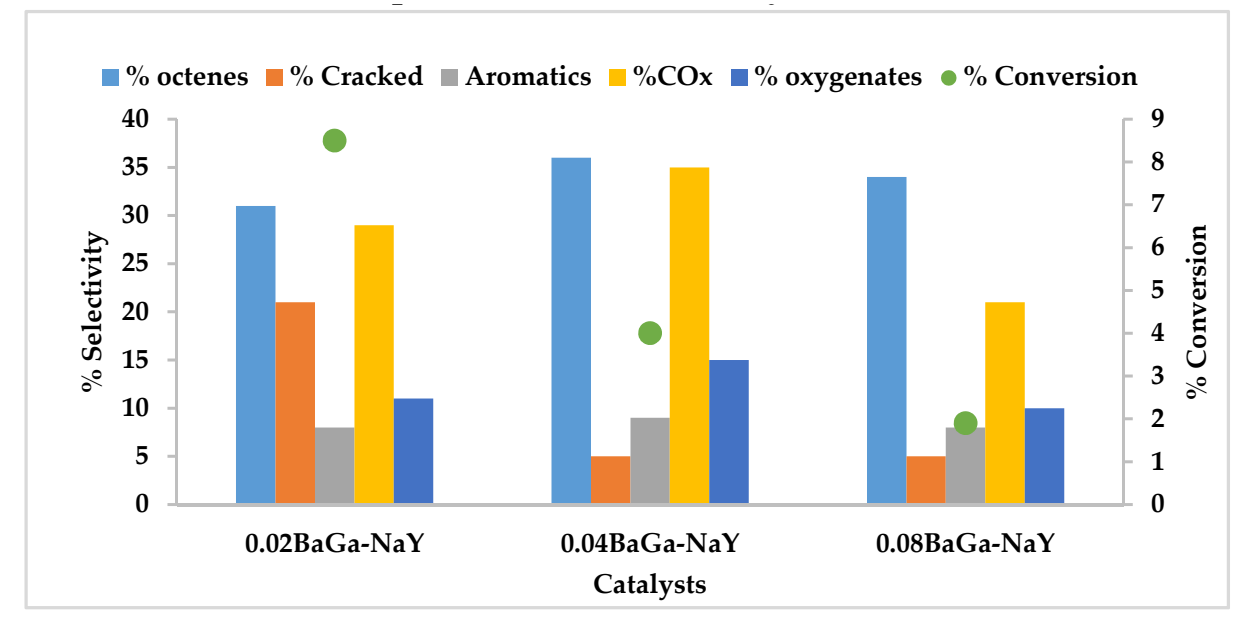

Figure 6. Effect of Ba metal content on the conversion of $n$-octane and selectivity towards cracked products, aromatics, carbon oxides, oxygenates and octene isomers at a temperature of $450{ }^{\circ} \mathrm{C}, \mathrm{C}: \mathrm{O}$ ratio of $8: 1$ with the GHSV fixed at $8000 \mathrm{~h}^{-1}$.

\subsubsection{Product Selectivity at iso-Conversion}

The activation of $n$-octane at $450{ }^{\circ} \mathrm{C}$, using the 0.04 mol alkaline earth metal modified Ga-NaY catalysts showed the previously reported ODH products of $n$-octane, which were octenes, aromatics, oxygenates, cracked products and carbon oxides [1-4]. Only GHSV was varied between the catalysts to achieve iso-conversion. Following the TPR and BET data trend, the catalysts with lower surface areas and which reduced at higher temperatures ( $\mathrm{SrGa}-\mathrm{NaY}$ and BaGa-NaY) were tested at $6000 \mathrm{~h}^{-1}$, while the other catalysts which reduced at lower temperatures (Ga-NaY, MgGa-NaY and $\mathrm{CaGa}-\mathrm{NaY}$ ) were 
tested at $8000 \mathrm{~h}^{-1}$. The conversions obtained over all the catalysts were $6 \pm 1 \%$ (Figure 7). The parent $\mathrm{NaY}$ catalyst was effectively inactive ( $2 \%$ conversion and $71 \%$ carbon balance) and was mostly selective to COx (51\%) and cracked products (19\%) and octene selectivity was $21 \%$. Amongst all the catalysts, $\mathrm{BaGa}-\mathrm{NaY}$ showed the highest selectivity to octenes, which can be explained by the catalyst's lowest acidity and highest medium-strong basic sites. The BaGa-NaY and SrGa-NaY catalysts also showed a reduced tendency to $\mathrm{COx}$ formation compared to the other three tested catalysts, and this low selectivity to COx and high octene selectivity confirms that indeed octenes are the precursors of COx, resulting from slow desorption of these secondary products from the less basic catalyst's surface. This selectivity behavior shown by the Ba and Sr modified catalysts may be explained by the high reduction temperature profiles and low degree of reducibility of the two catalysts. Ga-NaY, $\mathrm{CaGa-NaY}$ and MgGa-NaY showed two reduction peaks with a higher degree of reducibility, which suggests a reduction of $\mathrm{Ga}$ to its +1 oxidation state. This oxidation state is a more active state and might contribute to the over-oxidation of octenes to COx. Also, the high concentration of strong basic sites on BaGa-NaY and SrGa-NaY would assist the rapid desorption of the basic octenes. The cracked products selectivity was highest for the catalyst with the highest degree of acidity ( $\mathrm{CaGa}-\mathrm{NaY})$. The acid sites responsible for the formation of cracked products are known to be the Brønsted acid sites. With the prepared catalysts, this suggests that the responsible acid sites are the medium strength Brønsted acid sites since the $\mathrm{NH}_{3}$-TPD data shows that the catalysts with high cracked product selectivity (Ga-NaY and $\mathrm{CaGa}-\mathrm{NaY}$ ) have the highest density of medium strength acid sites. The other three ( $\mathrm{Mg}$, $\mathrm{Sr}$, and $\mathrm{Ba})$ catalysts have Lewis acid sites in the strongest acid sites region (above $400^{\circ} \mathrm{C}$ ) of the TPD which are responsible for the dehydrogenation products. These results are supported by the pyridine-IR spectra carried out after evacuation at $200^{\circ} \mathrm{C}$, where only Ga-NaY and CaGa-NaY still showed the Brønsted acid site bands, while the $\mathrm{Mg}$, $\mathrm{Sr}$, and Ba modified zeolites showed only the Lewis acid sites bands.

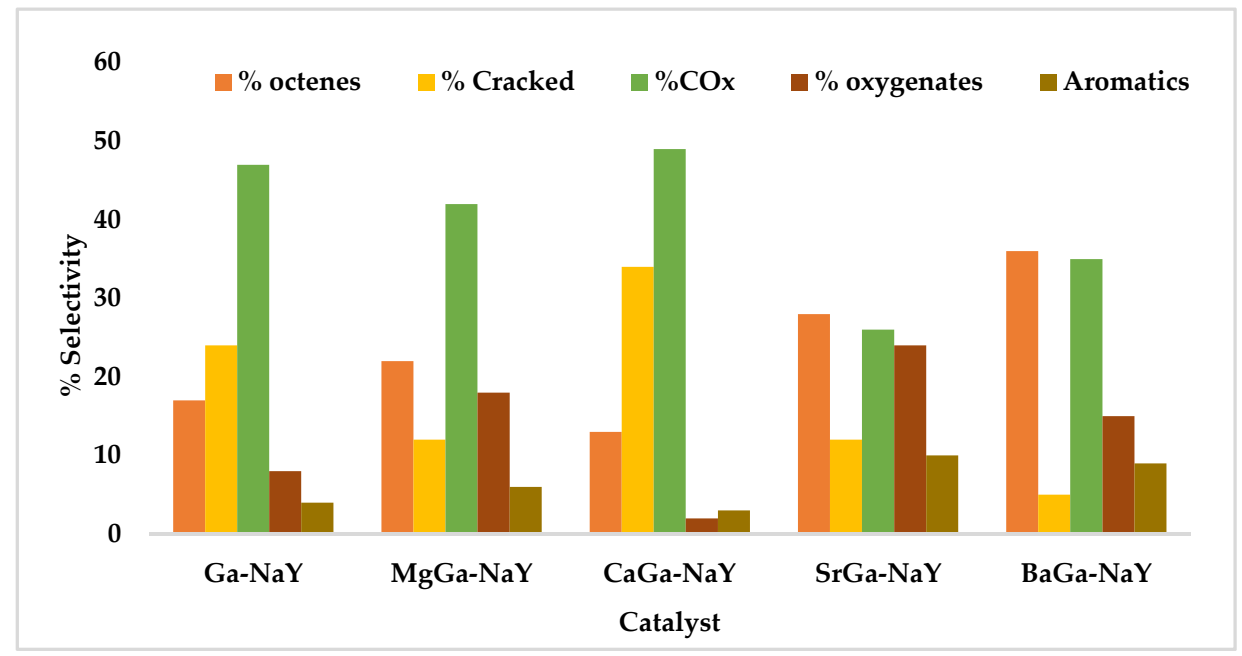

Figure 7. Products selectivity towards cracked products, aromatics, carbon oxides, oxygenates and octene isomers at iso-conversion $(6 \pm 1 \%)$ of $n$-octane at a fixed temperature of $450{ }^{\circ} \mathrm{C}$.

Figure 8 shows the cracked products (alkenes) distribution over the alkaline earth metal modified catalysts. The most dominant products for the two most acidic catalysts (Ga-NaY and $\mathrm{CaGa-NaY)}$ were the short chain alkenes, ethene, and propene, with the latter dominating. The selectivity to medium chain alkenes (pentene and hexene) increases with the basicity of the catalysts, with the Ba modified catalyst having the highest selectivity to pentene and hexene. The ratio of Lewis acid sites to Brønsted acid sites determines the ratio of alkene to alkane formation for the cracked products. Based on the characterization presented (pyridine-IR and $\mathrm{NH}_{3}-\mathrm{TPD}$ ), it is shown that Lewis acid sites are more pronounced for the alkaline earth metal modified catalysts. The formation of pentene and hexene over the more basic catalysts $(\mathrm{Mg}, \mathrm{Sr}$, and $\mathrm{Ba}$ ) may be because the pentyl and hexyl radicals formed over 
these catalysts are not further cracked into even smaller alkanes, but instead are converted into alkenes following the strength and density of Lewis sites in these catalysts.

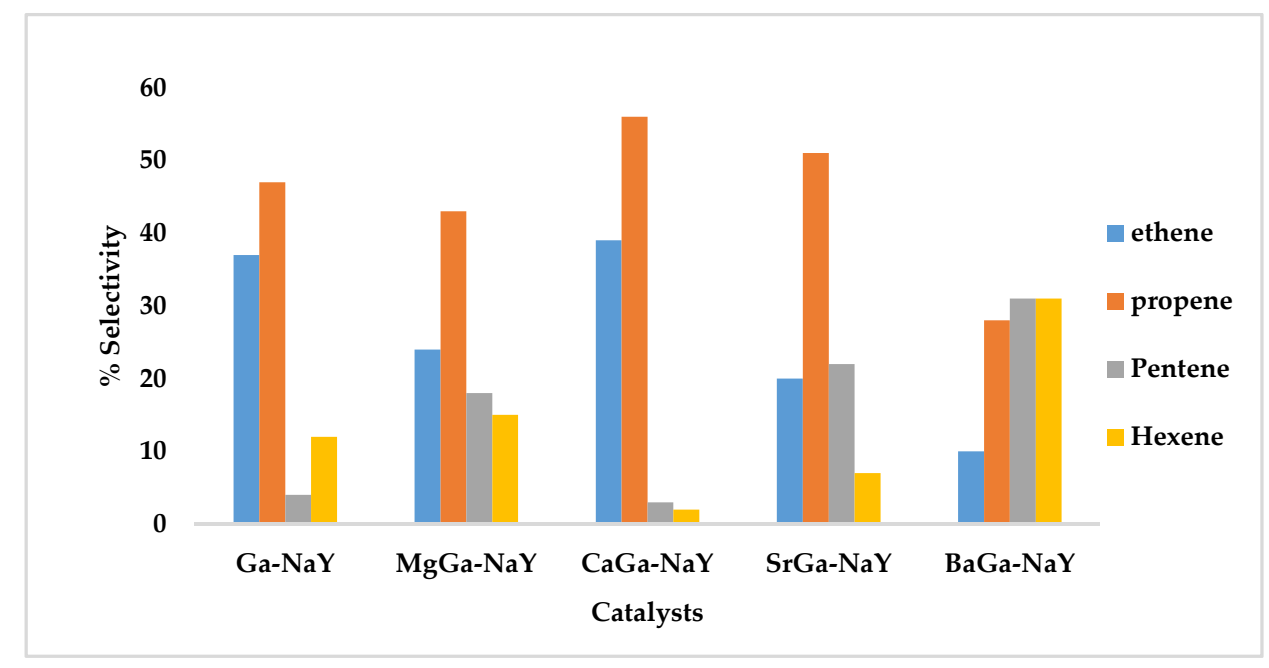

Figure 8. Selectivity towards different cracked products, at iso-conversion (6 $\pm 1 \%)$ of $n$-octane at a fixed temperature of $450^{\circ} \mathrm{C}$.

\section{Materials and Experimental Methods}

\subsection{Catalysts Preparation}

The Ga-NaY zeolite was prepared by a modified ionic exchanged procedure [22], where a solution of $2 \mathrm{~g} \mathrm{Ga}\left(\mathrm{NO}_{3}\right)_{3}$ (Sigma Aldrich, St. Louis, M.O., USA) in $15 \mathrm{~mL}$ of distilled water was mixed with $54 \mathrm{~g}$ of $\mathrm{NaY}$ (Sigma Aldrich-Si/Al = 2,6, St. Louis, M.O., USA) and stirred at $80^{\circ} \mathrm{C}$ for four hours. Thereafter, the contents were cooled to room temperature, filtered under vacuum and washed thoroughly with double distilled water before drying at $110^{\circ} \mathrm{C}$ overnight. The ionic exchange procedure was carried out twice to ensure sufficient loading of gallium on the zeolite.

Alkali earth metal $\left(\mathrm{M}=\mathrm{MgCl}_{2}, \mathrm{CaCl}_{2}, \mathrm{SrCl}_{2}, \mathrm{BaCl}_{2}\right)$ loaded $\mathrm{Ga}-\mathrm{NaY}$ zeolites were prepared by exchanging a $0.04 \mathrm{~mol}$ solution of alkali earth metal with $8 \mathrm{~g}$ of $\mathrm{Ga}-\mathrm{NaY}$ at $80^{\circ} \mathrm{C}$ for four hours. For Ba loaded Ga-NaY, two more catalysts were prepared with $0.02 \mathrm{~mol}$ and $0.08 \mathrm{~mol}$ of Ba solution. The resulting catalysts were filtered, washed and dried the same way as Ga-NaY. All the prepared catalysts were calcined under flowing air at $550{ }^{\circ} \mathrm{C}$ for six hours before characterization and testing. The prepared catalysts were coded as 0.02BaGa-NaY, BaGa-NaY, 0.08Ba-GaNaY, MgGa-NaY, CaGa-NaY and $\mathrm{SrGa}-\mathrm{NaY}$, where 0.02 and 0.08 referred to the number of moles of $\mathrm{Ba}$ in a catalyst. The catalysts without units before the name contain 0.04 moles of the alkaline earth metal. MGa-NaY implies that M and $\mathrm{Ga}$ are both introduced to $\mathrm{NaY}$ using ionic exchange.

\subsection{Catalysts Characterization}

Powder X-ray diffraction patterns for structure and phase identification were carried out using a Bruker D8 advance diffractometer equipped with a graphite monochromatic filter operated at $40 \mathrm{kV}$ and $40 \mathrm{~mA}$, (Billerica, M.A., USA). The radiation source was $\mathrm{CuK} \alpha$ with a $\lambda$ of $1.5406 \mathrm{~nm}$. The data collection was done at a step and scanning speed of $0.02^{\circ}$ and $0.2 \mathrm{~s}^{-1}$, respectively, and at a 2-theta range of $5^{\circ}$ to $90^{\circ}$. Inductively coupled plasma optical emission spectroscopy (ICP-OES) was performed to quantify the elemental composition of the material using a Perkin Elmer Optima 5300 DV spectrometer, (Waltham, M.A., USA). Standards of 1000 ppm Ga, Na, Mg, Ca, Sr, and Ba were all purchased from Fluka (Canterbury, UK). $\mathrm{N}_{2}$ physisorption analysis was performed using a Micromeritics Tristar II Surface area and porosity analyser, (Untershleissheim, Germany) operated at $-196^{\circ} \mathrm{C}$. Finely ground samples were degassed for one hour at $90^{\circ} \mathrm{C}$, then $200{ }^{\circ} \mathrm{C}$ for another hour and then at $400{ }^{\circ} \mathrm{C}$ for $6 \mathrm{~h}$ 
under flow of $\mathrm{N}_{2}$ before analysis, using a Micromeritics Flow Prep 060 instrument (New Jersey, N.J., USA). A Micromeritics 2920 Autochem II Chemisorption Analyser (Norcross, G.A., USA) was used for all temperature programmed experiments. Temperature programmed reduction (TPR) profiles were obtained by a published procedure. [2] Ammonia temperature programmed desorption $\left(\mathrm{NH}_{3}-\mathrm{TPD}\right)$ experiments were carried out using about $0.06 \mathrm{~g}$ of catalysts by a reported procedure [4]. $\mathrm{CO}_{2}-\mathrm{TPD}$ was carried out using $0.06 \mathrm{~g}$ of catalyst. The sample was heated up to $400{ }^{\circ} \mathrm{C}$ under a stream of helium (30 mL/min) for 30 minutes, followed by a temperature decrease to $80^{\circ} \mathrm{C}$ under the same stream of helium before exposing the sample to carbon dioxide. The TPD tests were carried out by heating the sample at $10{ }^{\circ} \mathrm{C} / \mathrm{min}$ until $900^{\circ} \mathrm{C}$ in a constant He flow. Infrared spectra were recorded at room temperature using a Perkin Elmer Spectrum 100 FT-IR Spectrometer equipped with a Universal ATR Sampling Accessory. Pyridine IR spectra were collected using the same equipment. For pyridine IR, $0.5 \mathrm{~g}$ samples were treated with $1.0 \mathrm{~mL}$ of liquid pyridine. After 3 hours, the samples were heated to $100^{\circ} \mathrm{C}$ and then $200^{\circ} \mathrm{C}$ under vacuum. The samples were cooled to room temperature before collecting the spectra in the region of $1400-1700 \mathrm{~cm}^{-1}$. For morphology and surface structure, SEM-EDX images were obtained using a Zeiss Ultra Plus field emission gun scanning electron microscope (FEG-SEM) with Smart SEM Software (Oberkhochen, Germany). Before analysis, the samples were coated with gold using a Q150R series high vacuum Quorum sputter coater (Laughton, UK).

\subsection{Catalytic Testing}

Reactions were carried out in a laboratory scale continuous-flow fixed-bed, gas phase reactor at the temperature of $450{ }^{\circ} \mathrm{C}$. Air and $\mathrm{N}_{2}$ were delivered to the reactor as the oxidant and diluent gas respectively. Reactions were performed with $n$-octane from Merck (New Jersey, N.J., USA) with a purity $>98 \%$, and concentration in a gaseous mixture (v/v) above the octane's upper flammability limit. A calibrated Lab Alliance Series II HPLC Pump, (New York, N.Y., USA) was used to feed $n$-octane into the system and the mass delivered was weighed using an electronic balance. The $n$-octane fed was maintained in the gaseous phase by heated feed lines at $130{ }^{\circ} \mathrm{C}$ using heating tape. The temperature was controlled using a CB-100 RK temperature control unit with an internal replay and monitored using K-type thermocouples. All the reactions were carried out using $1.0 \mathrm{~mL}$ of a pelletized catalyst in between two thin layers of glass wool positioned at the hottest zone of the calibrated reactor block. The spaces in the reactor tube were packed with 24 gritt carborundum and stoppered by glass-wool on both ends. Total gas flow was measured using a Ritter drum type wet gas flow meter (Bochum, Germany). All liquid products and unreacted feed were collected in a cylindrical stainless-steel vessel, cooled to $\approx 3.0^{\circ} \mathrm{C}$. A Perkin Elmer Clarus 400 GC (Waltham, M.A., USA) fitted with a $30 \mathrm{~m} \times 530 \mu \mathrm{m}$ Supelco Carboxen 106 PLOT column and a thermal conductivity detector was used to analyse COx products. Liquid and gaseous products were analysed using a Shimadzu GC-2025 (Kyoto, Japan), fitted with a $50 \mathrm{~m} \times 200 \mu \mathrm{m}$ PONA capillary column and flame ionization detector. All the data points were obtained in triplicate with carbon balances ranging from $97 \%$ to $100 \%$ and error of $\pm 2 \%$.

\section{Conclusions}

This study showed that $\mathrm{Ga}-\mathrm{NaY}$ modification with alkaline earth metals ( $\mathrm{Mg}, \mathrm{Ca}, \mathrm{Sr}$, and $\mathrm{Ba}$ ) can be carried out using the ionic exchange procedure, with the maintenance of the framework structure of faujasite with little or no crystallinity loss. This was shown using powder XRD, and the incorporation of the metals was shown using ICP-OES. The optimum alkaline earth metal loading was found to be $1.5 \mathrm{wt} \%$ for a $1 \mathrm{wt} \% \mathrm{Ga}-\mathrm{NaY}$ catalyst. Alkaline earth metals exchanged into the faujasite zeolite reduced the strength and amount of Brønsted acid sites of the zeolite, while increasing the basicity of the catalyst. However, Ca modified Ga-NaY, because of its hygroscopic nature, forms the acidic hydroxyl group, which increases the Brønsted acidity of the catalysts. This was consistent in all the characterizations (pyridine-IR, $\mathrm{NH}_{3}-\mathrm{TPD}, \mathrm{CO}_{2}$-TPD) used in this study.

Catalytic data obtained showed that active metal reducibility is proportional to the activity of the catalyst. BaGa-NaY, which was the least reducible catalyst, had the least activity. $\mathrm{Ga}^{+}$was found to be 
more active than $\mathrm{Ga}^{2+}$. The selectivity of the prepared catalysts was associated with the reducibility and basicity, which was affected by the alkaline earth metals. Brønsted acidity for the catalysts was proportional to COx and cracked products formation, while Lewis acidity favored the formation of alkenes. The catalysts with more strong Lewis acid sites also favored the formation of medium chain alkenes, compared to the shorter chain alkanes and alkenes favored by the catalysts with more Brønsted acid sites. The most basic barium modified Ga-NaY had the highest selectivity to octenes and low selectivity to COx and cracked products. The alkaline earth metal modified catalysts showed no sign of coking and maintained their activity for over three days. However, this was not the case for unmodified Ga-NaY and CaGa-NaY, and the coking of the two catalysts was due to the density of medium strength acid sites which adsorbed the alkenes and facilitated secondary reactions leading to coke formation. Some results obtained in previous studies using other systems such as vanadium oxides and platinum catalysts showed higher activity compared to the results obtained in this study. This suggest that the system used in this study cannot, presently, be thought of as a potential replacement for the DH catalysts used industrially. However, this study opens a door for future studies with the aim of improving the zeolites systems to use in ODH reactions, as the system offers a material that is easy to synthesize and modify, with interesting properties, that leads to catalysts with much longer lifetime compared to the metal oxides catalysts.

Supplementary Materials: The following are available online at http://www.mdpi.com/2073-4344/10/4/363/s1, Figure S1: SEM micrographs of (A) NaY, (B) Ga-NaY, (C) 0.02BaGa-NaY, (D) MgGa-NaY, (E) CaGa-NaY, (F) SrGa-NaY, (G) BaGa-NaY, (H) 0.08BaGa-NaY; Figure S2: SEM-EDX micrographs of (A) 0.02BaGa-NaY, (B) BaGa-NaY, (C) 0.08BaGa-NaY; Figure S3: NH3-TPD Profiles of (A) NaY, (B) Ga-NaY, (C) MgGa-NaY, (D) SrGa-NaY, (E) BaGa-NaY, (F) CaGa-NaY; Table S1: Temperature programmed reduction results for the Ga-NaY zeolites.

Author Contributions: Synthesis, Characterization, catalysis measurements: S.S.N.; data analysis: S.S.N., H.B.F. and M.N.C.; project supervision and manuscript preparation: H.B.F. and M.N.C. All authors read and agreed to the published version of the manuscript.

Funding: This research was funded by $C^{*}$ Change, the DST-NRF Centre of Excellence in catalysis.

Acknowledgments: We would like to thank the School of Chemistry and Physics, Microscopy and Microanalysis Unit (UKZN) for characterization and M. Datt (Sasol) for constructive criticism for this work.

Conflicts of Interest: The authors declare no conflicts of interest.

\section{References}

1. Farahani, M.D.; Dasireddy, V.D.B.C.; Friedrich, H.B. Oxidative Dehydrogenation of $n$-Octane over Niobium-Doped $\mathrm{NiAl}_{2} \mathrm{O}_{4}$ : An Example of Beneficial Coking in Catalysis over Spinel. ChemCatChem 2018, 10, 2059-2069. [CrossRef]

2. Bandaru, H.; Mahomed, A.S.; Singh, S.; Friedrich, H.B. The Effect of Varying the Metal Ratio in Chromium Molybdate Catalysts for the Oxidative Dehydrogenation of $n$-Octane. Mol. Catal. 2018, 460, 74-82. [CrossRef]

3. Fadlalla, M.I.; Farahani, M.D.; Friedrich, H.B. Three Inter-linked Active Sites in the Dehydrogenation of $n$-Octane over Magnesium Molybdate Based Catalysts and their Influence on Coking and Cracking Side Reactions. Mol. Catal. 2018, 461, 86-96. [CrossRef]

4. Elkhalifa, E.A.; Friedrich, H.B. Effects of Boron and Barium Dopants on VMgO Catalysts Employed in the Oxidative Dehydrogenation of $n$-Octane. Kinet. Catal. 2015, 56, 212-221. [CrossRef]

5. Obligacion, J.V.; Chirik, P.J. Earth-abundant Transition Metal Catalysts for Alkene Hydrosilylation and Hydroboration. Nat. Rev. Chem. 2018, 2, 15-34. [CrossRef] [PubMed]

6. Maleki, A. Green Oxidation Protocol: Selective Conversions of Alcohols and Alkenes to Al- dehydes, Ketones and Epoxides by Using a New Multiwall Carbon Nanotube-based Hybrid Nanocatalyst via Ultrasound Irradiation. Ultrason. Sonochem. 2017, 40, 460-464. [CrossRef] [PubMed]

7. Franke, R.; Selent, D.; Börner, A. Applied Hydroformylation. Chem. Rev. 2012, 112, 5675-5732. [CrossRef]

8. Sattler, J.J.H.B.; Ruiz-Martinez, J.; Santillan-Jimenez, E.; Weckhuysen, B.M. Catalytic Dehydrogenation of Light Alkanes on Metals and Metal Oxides. Chem. Rev. 2014, 114, 10613-10653. [CrossRef]

9. Shi, L.; Wang, Y.; Yan, B.; Song, W.; Shao, D.; Lu, A. Progress in Selective Oxidative Dehydrogenation of Light Alkanes to Olefins Promoted by Boron Nitride Catalysts. Chem. Commun. 2018, 54, 10936. [CrossRef] 
10. Yun, Y.S.; Lee, M.; Sung, J.; Yun, D.; Kim, T.Y.; Park, H.; Lee, K.R.; Song, C.K.; Kim, Y.; Lee, J.; et al. Promoting Effect of Cerium on MoVTeNb Mixed Oxide Catalyst for Oxidative Dehydrogenation of Ethane to Ethylene. Appl. Catal. B. 2018, 237, 554-562. [CrossRef]

11. Grabowski, R. Kinetics of oxidative dehydrogenation of C2-C3 alkanes on oxide catalysts. Catal. Rev. 2007, 48, 199-268. [CrossRef]

12. Gaab, S.; Machli, M.; Grasselli, R.K.; Lercher, J.A. Oxidative Dehydrogenation of Ethane over Novel Li/Dy/Mg Mixed Oxides: Structure-activity Study. Top. Catal. 2003, 23, 151-158. [CrossRef]

13. Madeira, L.M.; Martìn-Aranda, R.M.; Maldonado-Hódar, F.J.; Fierro, J.L.G.; Portela, M.F. Oxidative Dehydrogenation of $n$-Butane over Alkali and Alkaline Earth-Promoted $\alpha-\mathrm{NiMoO}_{4}$ Catalysts. J. Catal. 1997, 169, 469-479. [CrossRef]

14. Mulla, S.A.R.; Choudhary, V.R. Oxidative Conversion of Ethane to Ethylene over Supported SrO-Promoted $\mathrm{Er}_{2} \mathrm{O}_{3}$ Catalyst. J. Mol. Catal. A Chem. 2004, 223, 259-262. [CrossRef]

15. Maiti, A.; Govind, N.; Kung p King-Smith, D.; Miller, J.E. Effect of Surface Phosphorus on the Oxidative Dehydrogenation of Ethane: A First-Principles Investigation. J. Chem. Phys. 2002, 117, 8080-8088. [CrossRef]

16. Dai, H.X.; Ng, C.F.; Au, C.T. SrCl2-promoted REOx ( $\mathrm{RE}=\mathrm{Ce}, \mathrm{Pr}$, Tb) catalysts for the selective oxidation of ethane: A study on performance and defect structures for ethene formation. J. Catal. 2001, 199, 177-192. [CrossRef]

17. Gounden, N.; Friedrich, H.B.; Mahadevaiah, N.; Fadlalla, M.I. Octenes and Aromatics from the Oxidative Dehydrogenation of $n$-Octane over $\mathrm{Co} / \mathrm{TiO}_{2}$ Catalysts. Catal. Lett. 2014, 144, 2043-2051. [CrossRef]

18. Elkhalifa, E.A.; Friedrich, H.B. Oxidative Dehydrogenation and Aromatization of $n$-Octane over $\mathrm{VMgO}$ Catalysts Obtained by Using Different MgO Precursors and Different Precursor Treatments. J. Mol. Catal. A Chem. 2014, 392, 22-30. [CrossRef]

19. Vedrine, J.C. Heterogeneous Catalytic Partial Oxidation of Lower Alkanes (C1-C6) on Mixed Metal Oxides. J. Energy Chem. 2016, 25, 936-946. [CrossRef]

20. Atanga, M.A.; Rezaei, F.; Jawad, A.; Fitch, M.; Rownaghi, A.A. Oxidative Dehydrogenation of Propane to Propylene with Carbon Dioxide. Appl. Catal. B. 2018, 220, 429-445. [CrossRef]

21. Cheng, Y.; Zhang, F.; Zhang, Y.; Miao, C.; Hua, W.; Yue, Y.; Gao, Z. Oxidative Dehydrogenation of Ethane With $\mathrm{CO}_{2}$ over $\mathrm{Cr}$ Supported on Submicron ZSM-5 Zeolite. Chin. J. Catal. 2015, 36, 1242-1248. [CrossRef]

22. Lin, X.; Hoel, C.A.; Sachtler, W.M.H.; Poeppelmeier, K.R.; Weitz, E. Oxidative Dehydrogenation (ODH) of Ethane With $\mathrm{O}_{2}$ as Oxidant on Selected Transition Metal-Loaded Zeolites. J. Catal. 2009, 265, 54-62. [CrossRef]

23. Cheng, Y.; Lei, T.; Miao, C.; Hua, W.; Yue, Y.; Gao, Z. $\mathrm{Ga}_{2} \mathrm{O}_{3} / \mathrm{NaZSM}-5$ for $\mathrm{C} 2 \mathrm{H} 6$ dehydrogenation in the presence of $\mathrm{CO}_{2}$ : Conjugated effect of silanol. Micropor. Mesopor. Mater. 2018, 268, 235-242. [CrossRef]

24. Richardson, J.T.; Scates, R.M.; Twigg, M.V. X-ray diffraction study of the hydrogen reduction of $\mathrm{NiO} / \alpha-\mathrm{Al}_{2} \mathrm{O}_{3}$ steam reforming catalysts. Appl. Catal. A Gen. 2004, 267, 35-46. [CrossRef]

25. Huang, Y.; Wang, K.; Dong, D.; Li, D.; Hill, M.R.; Hill, A.J.; Wang, H. Synthesis of Hierarchical Porous Zeolite NaY Particles with Controllable Particle Sizes. Micropor. Mesopor. Mater. 2010, 127, 167-175. [CrossRef]

26. Alwash, A.H.; Abdullah, A.Z.; Ismail, N. Elucidation of Reaction Behaviors in Sonocatalytic Decolorization of Amaranth Dye in Water Using Zeolite Y Co-incorporated with Fe and $\mathrm{TiO}_{2}$. Adv. Chem. Eng. Sci. 2013, 3, 113-122. [CrossRef]

27. Chanthaanont, P.; Sirivat, A. Effect of Transition Metal Ion-Exchanged into the Zeolite $\mathrm{Y}$ on Electrical Conductivity and Response of PEDOT-PSS/MY Composites toward $\mathrm{SO}_{2}$. Adv. Polym. Tech. 2013, 32, 21367-21372. [CrossRef]

28. Zhao, J.; Yin, Y.; Li, Y.; Chen, W.; Liu, B. Synthesis and Characterization of Mesoporous Zeolite Y by Using Block Copolymers as Templates. Chem. Eng. J. 2016, 284, 405-411. [CrossRef]

29. Pal, N.; Pramanik, M.; Bhaumik, A.; Ali, M. Highly Selective and Direct Oxidation of Cyclohexane to Cyclohexanone Over Vanadium Exchanged NaY at Room Temperature Under Solvent-Free Conditions. J. Mol. Catal. A Chem. 2014, 392, 299-307. [CrossRef]

30. Król, M.; Mozgawa, W.; Jastrzębski, W.; Barczyk, K. Application of IR Spectra in the Studies of Zeolites from D4R and D6R Structural Groups. Micropor. Mesopor. Mater. 2012, 156, 181-188. [CrossRef]

31. Shariatinia, Z.; Bagherpour, A. Synthesis of Zeolite NaY and its Nanocomposites With Chitosan as Adsorbents for Lead(II) Removal From Aqueous Solution. Powder Technol. 2018, 388, 744-763. [CrossRef] 
32. Datka, J.; Gil, B.; Baran, P. Heterogeneity of OH groups in HZSM-5 Zeolites: Splitting of OH and OD Bands in Low-Temperature IR Spectra. Micropor. Mesopor. Mater. 2003, 58, 291-294. [CrossRef]

33. Niwa, M.; Katada, N. New Method for the Temperature- Programmed Desorption (TPD) of Ammonia Experiment for Characterization of Zeolite Acidity: A Review. Chem. Rec. 2013, 13, 432-455. [CrossRef] [PubMed]

34. Brueva, T.R.; Mishin, I.V.; Kapustin, G.I. Distribution of Acid-site Strengths in Hydrogen Zeolites and Relationship Between Acidity and Aatalytic Activity. Thermochim. Acta 2001, 379, 15-23. [CrossRef]

35. Almutairi, S.M.T.; Mezari, B.; Filonenko, G.A.; Magusin, P.C.M.M.; Rigutto, M.S.; Pidko, E.A.; Hensen, E.J.M. Influence of Extraframework Aluminum on the Brønsted Acidity and Catalytic Reactivity of Faujasite Zeolite. ChemCatChem 2013, 5, 452-466. [CrossRef]

36. Jie, Y.; Dinghua, Y.; Peng, S.; He, H. Alkaline Earth Metal Modified NaY for Lactic Acid Dehydration to Acrylic Acid: Effect of Basic Sites on the Catalytic Performance. Chin. J. Catal. 2011, 32, 405-411.

37. Liu, C.; Aika, K. Ammonia Adsorption on Ion Exchanged Y-zeolites as Ammonia Storage Material. J. Jpn. Petrol. Inst. 2003, 46, 301-307. [CrossRef]

38. Choi, S.W.; Kim, W.G.; So, J.S.; Moore, J.S.; Liu, Y.J.; Dixit, R.S.; Pendergast, J.G.; Sievers, C.; Sholl, D.S.; Nair, S.; et al. Propane dehydrogenation catalyzed by gallosilicate MFI zeolites with perturbed acidity. J. Catal. 2017, 345, 113-123. [CrossRef]

39. Ates, A.; Akgül, G. Modification of natural zeolite with $\mathrm{NaOH}$ for removal of manganese in drinking water. Powder Technol. 2016, 287, 285-291. [CrossRef]

40. Xue, L.; He, H.; Liu, C.; Zhang, C.; Zhang, B. Promotion Effects and Mechanism of Alkali Metals and Alkaline Earth Metals on Cobalt-Cerium Composite Oxide Catalysts for $\mathrm{N}_{2} \mathrm{O}$ Decomposition. Environ. Sci. Tech. 2009, 43, 890-895. [CrossRef]

41. Zhong, C.; Guo, X.; Mao, D.; Wang, S.; Wu, G.; Lu, G. Effects of Alkaline-Earth Oxides on the Performance of a CuO-Z $\mathrm{ZrO}_{2}$ Catalyst for Methanol Synthesis via $\mathrm{CO}_{2}$ Hydrogenation. RSC Adv. 2015, 5, 52958-52965. [CrossRef]

42. Shao, C.; Lang, W.; Yan, X.; Guo, Y. Catalytic Performance of Gallium Oxide Based-Catalysts for the Propane Dehydrogenation Reaction: Effects of Support and Loading Amount. RSC Adv. 2017, 7, 4710-4723. [CrossRef]

43. El-Malki, E.1.-M.; van Santen, R.A.; Sachtler, W.M.H. Introduction of Zn, Ga, and Fe into HZSM-5 Cavities by Sublimation: Identification of Acid Sites. J. Phys. Chem. B 1999, 103, 4611-4622. [CrossRef]

44. Price, G.L.; Kanazi, V. $\mathrm{Ga}_{2} \mathrm{O}_{3} / \mathrm{HZSM}-5$ Propane Aromatization Catalysts: Formation of Active Centers via Solid-State Reaction. J. Catal. 1990, 126, 267-278. [CrossRef]

45. Li, J.; Zhang, C.; Cheng, X.; Qing, M.; Xu, J.; Wu, B.; Yang, Y.; Li, Y. Effects of Alkaline-Earth Metals on the Structure, Adsorption and Catalytic Behaviour of Iron-based Fischer-Tropsch Synthesis Catalysts. Appl. Catal. A Gen. 2013, 464-465, 10-19.

46. Homeyer, S.T.; Sachtler, W.M.H. Elementary Steps in the Formation of Highly Dispersed Palladium in NaY. J. Catal. 1989, 118, 266-274. [CrossRef]

(C) 2020 by the authors. Licensee MDPI, Basel, Switzerland. This article is an open access article distributed under the terms and conditions of the Creative Commons Attribution (CC BY) license (http://creativecommons.org/licenses/by/4.0/). 